\title{
Thermal Buckling of Metal Foam Sandwich Panels for Convective Thermal Protection Systems
}

\author{
Joseph F. Rakow* and Anthony M. Waas ${ }^{\dagger}$ \\ University of Michigan, Ann Arbor, Michigan 48109
}

\begin{abstract}
Sandwich panels with metal foam cores are studied with application to actively cooled thermal protection systems. To evaluate these panels under thermal loading, a novel experimental technique and load frame, which provide a prominent improvement in the simultaneous preservation of thermal and mechanical boundary conditions during thermomechanical structural testing, are introduced and validated. With this technique, the response of metal foam sandwich panels (MFSPs) to thermally induced in-plane equibiaxial loading is investigated, and the elastoplastic pre- and postbuckling response of MFSPs is measured and analyzed. The in-plane response of the panels is quantified with strain-gauge measurements, and the out-of-plane response across the surface of the panel is captured via shadow moire interferometry. These measurements provide direct visualization and quantification of the initial buckled mode shapes, as well as the evolution of the elastoplastic postbuckled mode shapes from initial buckling into the postbuckling regime. This experimental investigation is the first of its kind, complementing the largely theoretical and numerical body of information on the thermomechanical response of sandwich panels.
\end{abstract}

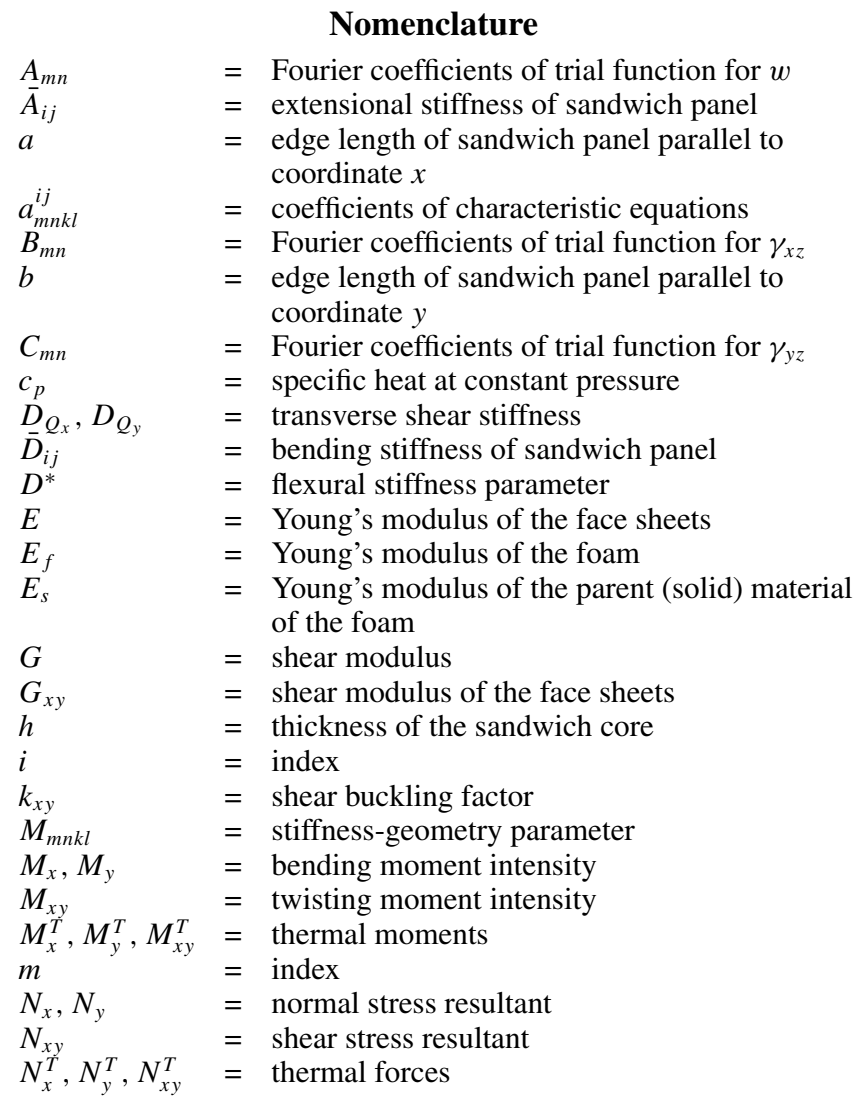

Received 2 April 2004; presented as Paper 2004-1710 at the AIAA/ASCE/ AHS/ASC 45th Structures, Structural Dynamics, and Materials Conference, Palm Springs, CA, 19-21 April 2004; revision received 1 September 2004; accepted for publication 17 October 2004. Copyright (C) 2004 by Joseph F. Rakow and Anthony M. Waas. Published by the American Institute of Aeronautics and Astronautics, Inc., with permission. Copies of this paper may be made for personal or internal use, on condition that the copier pay the $\$ 10.00$ per-copy fee to the Copyright Clearance Center, Inc., 222 Rosewood Drive, Danvers, MA 01923; include the code 0022-4650/05 \$10.00 in correspondence with the CCC.

${ }^{*}$ Graduate Student Research Assistant, Aerospace Engineering Department, 1320 Beal Street; jrakow@umich.edu. Student Member AIAA.

${ }^{\dagger}$ Professor, Aerospace Engineering Department, 1320 Beal Street; dcw@umich.edu. Associate Fellow AIAA.

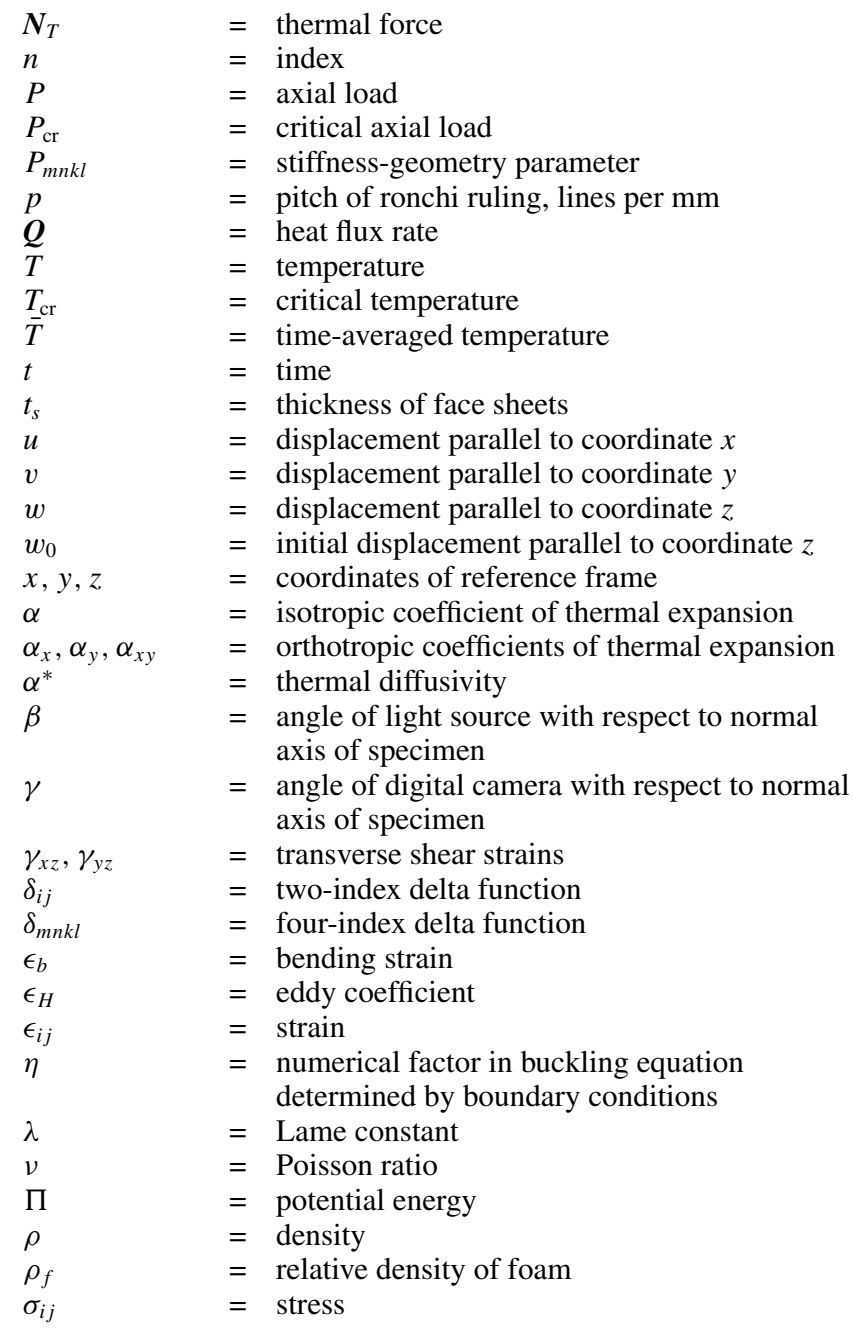

\section{Introduction}

$\mathbf{T}$ HERMAL protection during high-speed flight is arguably the most pressing issue in the advancement of hypersonic vehicles. Since the early 1950 s, with the development of the X-2 and $\mathrm{X}-15$ vehicles, designed to capitalize on the supersonic accomplishment of the X-1, until 2003, when the Space Shuttle Columbia 
disintegrated during hypersonic reentry, the technology to propel vehicles has been more mature and more successful than the technology to protect vehicles from the resulting aerodynamic heat loads. This limitation has manifested and dominated a variety of aspects of hypersonic vehicle operation, from flight planning, to vehicle maintenance, to flight performance envelopes. For example, with regard to flight planning, the ceramic thermal protection system (TPS) on the space shuttle cannot fly in wet weather because of its hygroscopic surface. With regard to vehicle maintenance, the space shuttle TPS, as a whole, dominates the maintenance requirements of the vehicle, demanding 32,000 (Ref. 1) work hours of inspection and repair required by the vehicle between flights. With regard to performance envelopes, on its record-setting flight above Mach 6, components of the X-15 were virtually destroyed when shock-shock interactions pierced holes in the inconel airframe, a heat sink TPS. Clearly, the current state of the art in TPS technology cannot efficiently manage the demanding requirements of hypersonic vehicle operation.

One concept for advanced structural TPSs is an actively cooled TPS, in which load-bearing structural members are integrated with coolant passages. ${ }^{2}$ Unlike the dual-component ablative TPS onboard the manned capsules of the Mercury, Gemini, and Apollo aircraft, and unlike the dual-component insulated system used on the space shuttle, the ideal structural TPS is a single-component multifunctional structure that bears both mechanical and thermal loads.

To this point, however, true integration and multifunctionality have not been achieved. An example of a first-generation actively cooled structural panel is shown in Fig. 1 (Ref. 3). This panel is a standard, honeycomb-cored sandwich panel that has been modified with a series of coolant tubes on the inside of the external face sheet. The problems associated with this type of construction are readily apparent:

1) The coolant tubes interrupt the path for shear load transfer between the face sheets and the honeycomb core.

2) Machining of the honeycomb and the layup of the panel to accommodate these tubes create significant manufacturing difficulties.

3) The discreet nature of the coolant passages causes severe thermal stress gradients throughout the panel.

4) Blockage in discretized coolant passages leads to local hot spots of reduced strength in the load-bearing structure.

For success, such problems must be overcome in next-generation actively cooled TPS concepts.

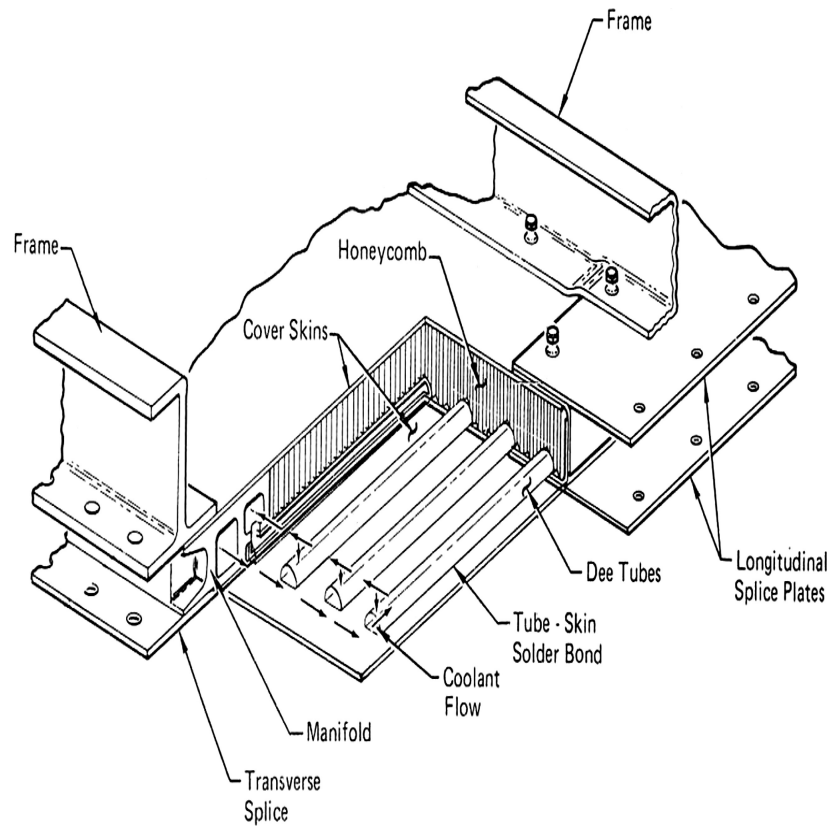

Fig. 1 First-generation actively cooled sandwich panel. ${ }^{3}$

\section{A. Improvements Offered by Metal Foam for TPSs}

Metal foam sandwich panels (MFSPs) provide a truly multifunctional structure that is well suited for the management of both thermal and mechanical loads in actively cooled TPSs. An actively cooled MFSP is a sandwich panel with an open-cell metal foam core integrally bonded, that is, brazed, to metal face sheets. Whereas the panel bears airframe flight loads, aerodynamic heat on the panel's outer surface is conducted through the outer face sheet and, with the assistance of the conductive foam, into coolant passing through the core of the panel (Fig. 2).

Metal foam is the key to the multifunctional panel, bearing mechanical shear loads while conductively distributing heat from the outer surface of the panel into and throughout the cross-sectional flow of the coolant within the panel. Metal foam, in its open-cell form, has a substructure similar to foam found in seat cushions and packing materials, but the substructure is made of metallic alloy, such as aluminum, titanium, inconel, or copper. Figure 3 shows an open-cell metal foam in detail. In previous studies, the present authors have demonstrated and characterized the response of metal foams under shear loading through experimentation, ${ }^{4}$ numerical simulation, ${ }^{5}$ and a micromechanics-based analysis. ${ }^{6}$

Metal foam is central to the improvements offered by MFSPs over previous actively cooled panel concepts:

1) The foam and face sheets provide a monolithic, single-material structure with no inherent intrastructural mismatch of coefficients of thermal expansion.

2) Through-the-thickness shear response and heat transfer can be controlled through the foam density.

3) The metal foam core provides a network of nondiscreet coolant passages, eliminating the severity of local coolant blockage.

4) With high internal surface area per unit volume $\left[\sim 1800\left(\mathrm{~m}^{2} / \mathrm{m}^{3}\right)\right]$, metal foam facilitates extremely high structurecoolant interaction per unit volume.

5) In addition to conducting heat, the foam core improves the heat transfer capability of the system by creating turbulence in the coolant, even at low Reynolds numbers. The departure from laminar flow introduces a nonzero eddy coefficient $\epsilon_{H}$ into the boundarylayer equations for steady, incompressible flow with constant properties, which increases the rate of heat flux $\boldsymbol{Q}$ to the coolant,

$$
\boldsymbol{Q}=-\rho c_{p}\left(\alpha^{*}+\epsilon_{H}\right) \frac{\partial \bar{T}}{\partial z}
$$

where $\alpha^{*}$ is the thermal diffusivity of the coolant and $\partial \bar{T} / \partial z$ is the time-averaged thermal gradient field perpendicular to the flow. ${ }^{7}$

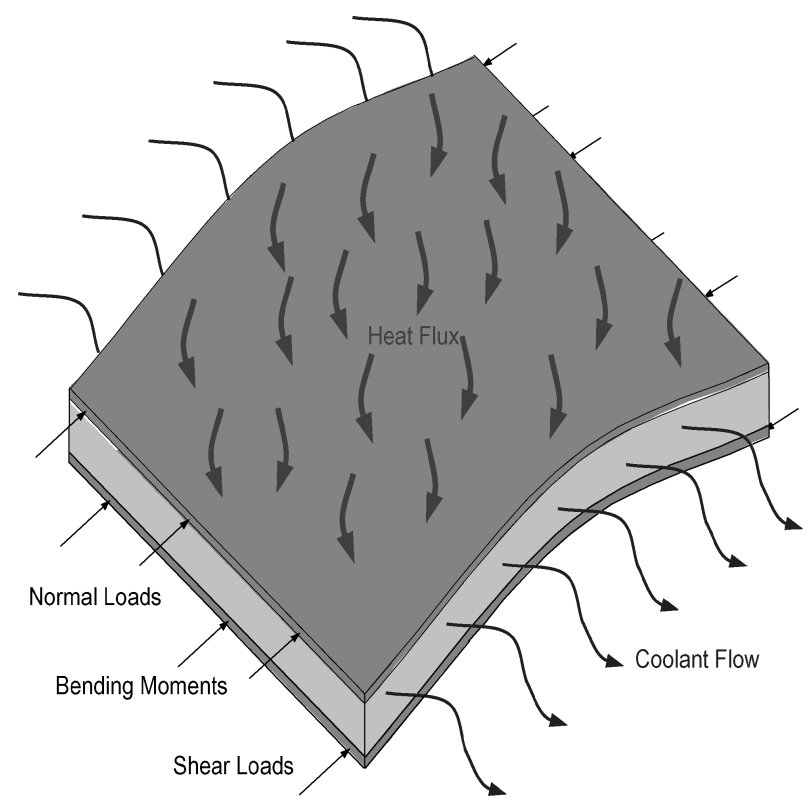

Fig. 2 Schematic of actively cooled MFSP. 

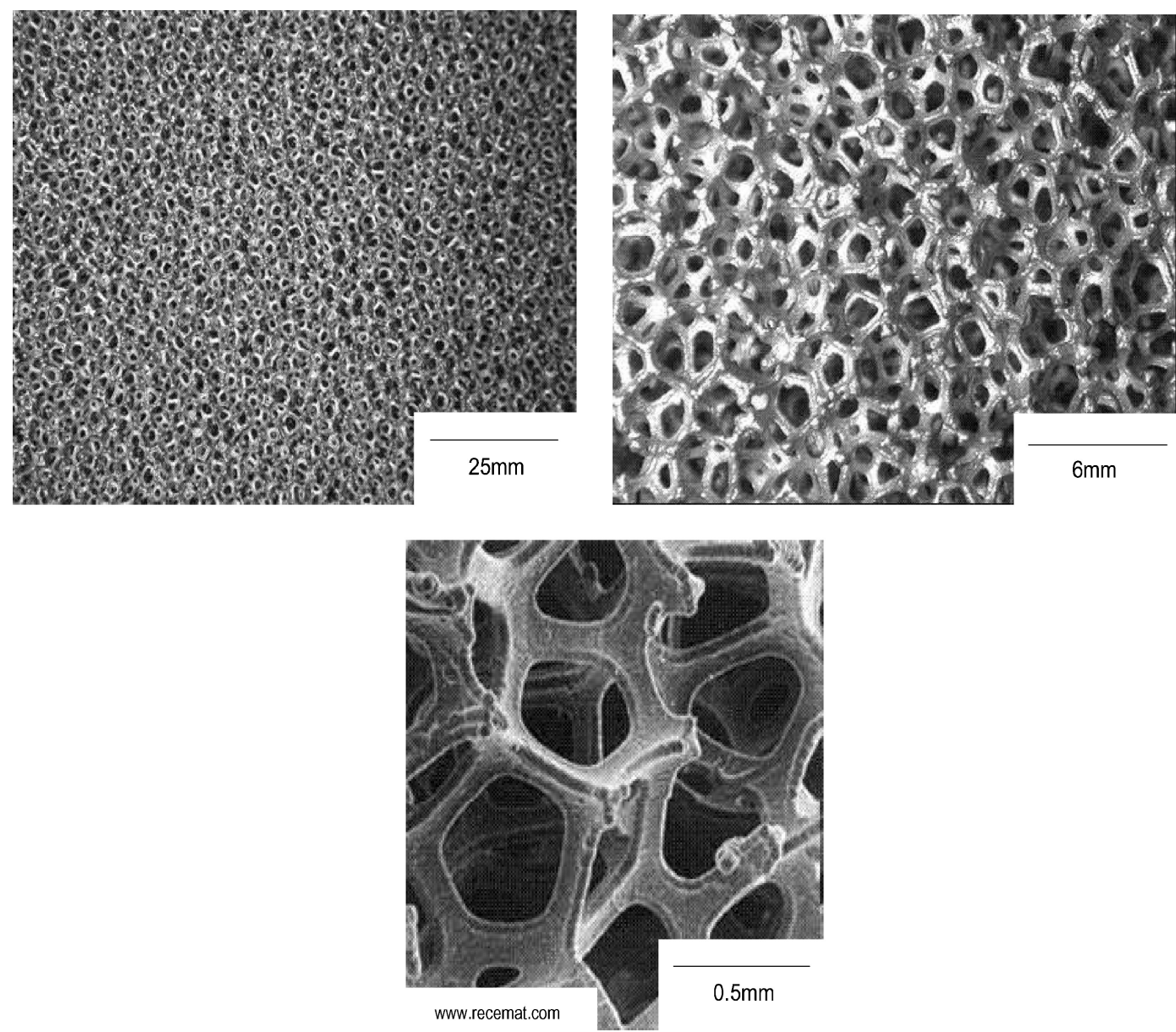

Fig. 3 Open-cell metal foam.

\section{B. Thermomechanical Response of MFSPs}

The response of MFSPs to in-plane thermomechanical loading is a driving design parameter for the integration of MFSPs into flightready hardware. Previous investigations into sandwich panels and other shear deformable panels under thermal loading are almost exclusively numerical and theoretical in nature ${ }^{8-13}$; very few efforts have analyzed the thermomechanical response of sandwich panels experimentally. Likewise, no data exist, in the authors' knowledge, for the thermomechanical response of MFSPs. The present work examines the response of MFSPs under quasistatic thermally induced equibiaxial compression loads, leading to buckling and elastoplastic postbuckling.

Section II of this paper describes the experimental procedure used in the present work to quantify the thermomechanical response of MFSPs under in-plane loading. Special attention is given to the experimental technique developed by the present authors to constrain and load panel-type structures without corrupting the thermal and mechanical boundary conditions in thermomechanical experiments. In common thermomechanical experimental methods, the thermal boundary conditions are corrupted by the presence of the mechanical boundary conditions, acting as a heat sink. ${ }^{14}$ This new method represents a departure from such complications. The validity of the technique is established by measuring the onset of thermal buckling in thin monolithic aluminum plates and comparing the results to classical thin-plate theory. The validated experimental technique is then utilized to subject MFSPs to thermally induced in-plane loading and to measure the response of the panels.

Section III presents the first experimental results on the thermal response and buckling of MFSPs. The in-plane response and the onset of out-of-plane deflections are indicated through strain-gauge measurements on the panel surfaces. The measurements provide the pre- and postbuckling response of MFSPs and identify the crit- ical temperature for the onset of thermal buckling in the panel. The out-of-plane postbuckling response, as well as the onset of thermal buckling, are captured by images of moire fringe patterns that quantify the evolution of the elastoplastic buckled mode shapes across the entire surface of the panel. The critical temperatures measured by each technique are compared to a theoretical prediction based on a Rayleigh-Ritz minimization of the potential energy of a shear deformable plate. The experimental and theoretical results are shown to be in very good agreement.

\section{Experimental Procedure}

\section{A. Experimental Load Frame}

Figure 4 shows the load frame designed by the present authors to induce in-plane thermal loading of MFSPs. The central concept of the frame is to develop in-plane loads in the specimen by mismatched coefficients of thermal expansion (CTE). An MFSP, with a specific CTE, is clamped in a frame made of a material with a CTE that is not equal to the CTE of the MFSP material, such as an aluminum MFSP in a steel frame, as is used in the present study. The frame-specimen assembly is heated uniformly. Through-the-thickness thermal gradients can also be applied and are currently in use by the present authors. Once heated, in-plane loads are created within the specimen because of the mismatched CTEs of the frame and specimen. With well-defined (measured) temperature-dependent CTEs and accurate measurements of the temperature throughout the assembly, the in-plane loading is well defined and given by

$$
N_{T}=\int \frac{E(z, T)\left[\alpha_{\text {frame }}(z, T)-\alpha_{\text {panel }}(z, T)\right] \Delta T(z)}{1-v(z, T)} \mathrm{d} z
$$

in which $\boldsymbol{N}_{T}$ is the in-plane load per unit edge length and $E=E_{\text {panel }}$ and $v=v_{\text {panel }}$. 


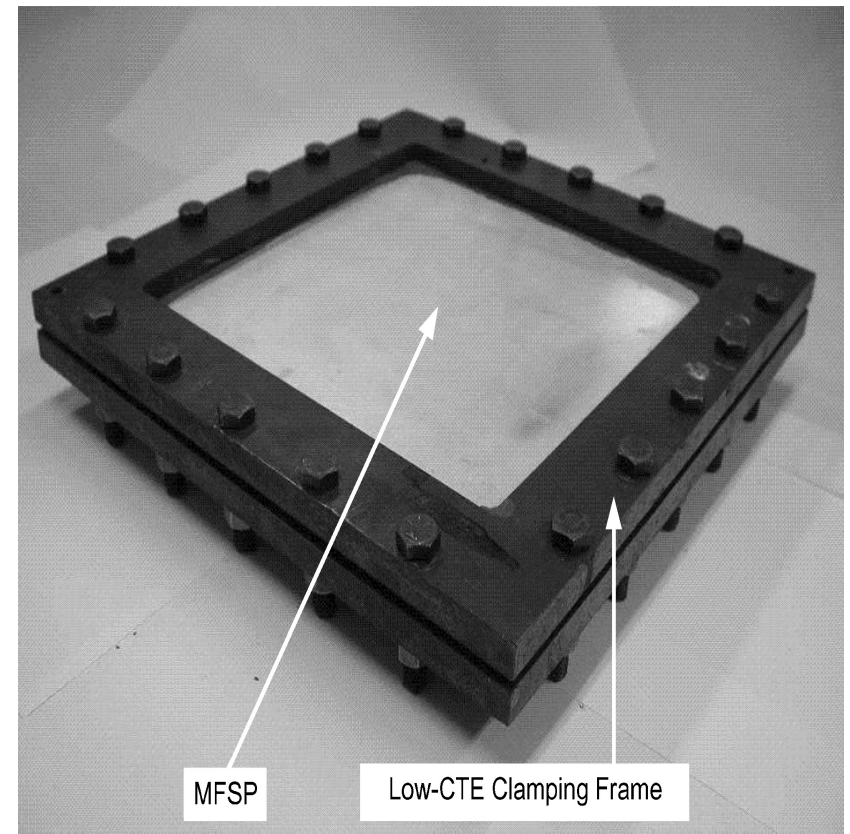

Fig. 4 Load frame used to clamp boundary of MFSP and induce inplane thermal stresses.

In typical thermomechanical structural experiments, it is necessary to impart a specific temperature field or heat flux throughout the specimen. Mechanical loads must also be introduced, and this typically involves contact between a load frame and the boundary of the specimen. Once contact is introduced, however, the load frame becomes a heat sink and the prescribed temperature or heat flux field in the specimen is compromised. Likewise, the severity of the thermal loading can often degrade the quality of the mechanical boundary conditions.

This challenging and occasionally prohibitive conflict is explored in Ref. 14, in which the author of Ref. 14 cites the work of Ref. 15 as a classic example of the conflict of thermomechanical boundary conditions. The thermomechanical load frame of Ref. 15 is shown in Fig. 5. In the experiment, the boundary conditions were to be clamped and one side of the panel was to be heated by radiant lamps to produce a temperature gradient through the thickness of the panel. To minimize the conductive heat sink of the clamps, the authors provided clamping along the boundary through a series of bolts. However, the bolts were not sufficient to properly realize the intended clamped boundary conditions. The insufficient clamping bolts were subsequently reinforced by stiff angle beams. The stiff angle beams, however, shaded the boundary of the panel, producing nonuniform heating of the panel. To equilibrate the in-plane temperature distribution, heat lamps were placed along the edges of the panel. However, these lamps degraded the desired thermal gradient through the thickness of the panel. The thermomechanical boundary conditions were in direct, and possibly unavoidable, conflict with each other.

The load frame designed for the present investigation offers a reprieve from this classic conflict of thermomechanical boundary conditions. The primary reason for this improvement is that the boundary-condition hardware is subjected to the same temperature history as the specimen. This condition eliminates thermal gradients between the specimen and load frame and allows the temperature field of the specimen to remain uncorrupted. The mechanical boundary conditions and the applied load, Eq. (2), remain well defined throughout the experiment through measurement of the temperature-dependent material properties (CTE and elastic moduli) of both the specimen and the frame throughout the entire temperature range of the experiment. The method has been used for both uniform and through-the-thickness static temperature fields, but remains unproven for in-plane thermal gradients and transient thermal loading.

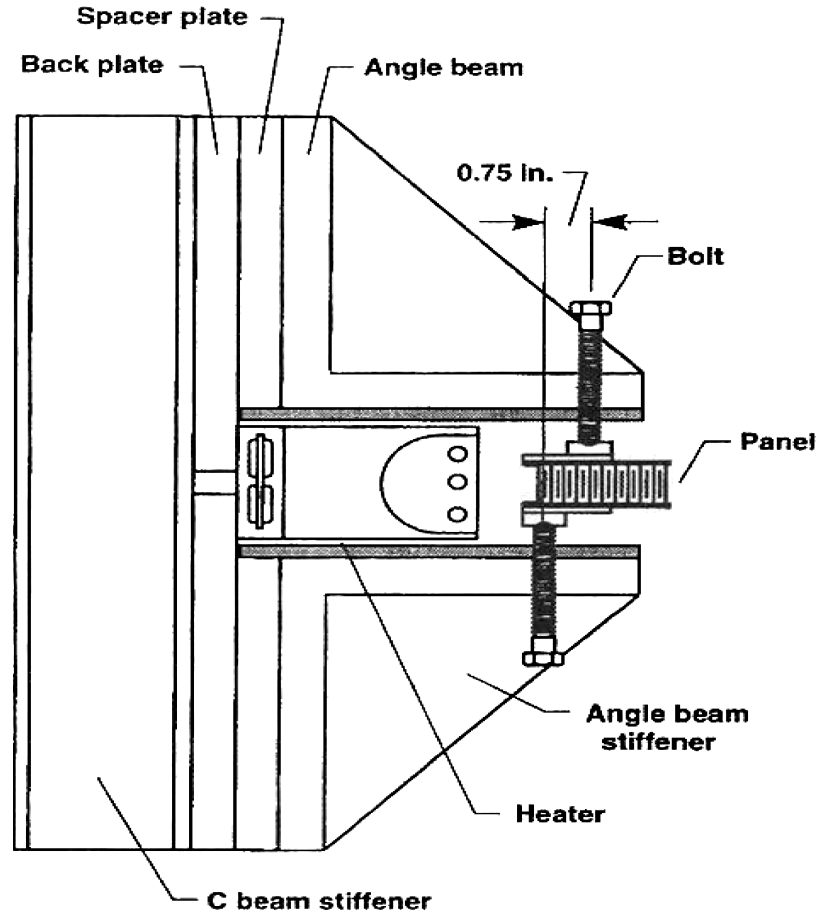

Fig. 5 Thermal structural load frame that demonstrates the conflicts typical of experimental thermomechanical boundary conditions.

Details of the frame are shown in Fig. 6. The frame has two identical pieces that are bolted together to provide a clamped boundary condition along the border of the MFSP. This leaves a central square test section measuring $216 \times 216 \mathrm{~mm}(8.5 \times 8.5 \mathrm{in}$. $)$. The transverse edges of the face sheets are in direct contact with the frame. Consistent contact along the entire perimeter of the panel-frame interface is ensured with a small bead of metallic putty in the panel-frame interface. The frame has been designed such that the foam core remains accessible for both the passage of coolant and for measurements within the core. Such necessities can be accommodated, because Young's modulus of the foam core is sufficiently small to justify the assumption that the contribution of the core to the in-plane loadbearing capability of the panel is negligible. The uniaxial stiffness of the foam $E_{f}$ scales as ${ }^{16}$

$$
E_{f}=(0.1-4.0) E_{s} \rho_{f}^{2}
$$

in which the factor $(0.1-4.0)$ represents a range of proportionality from 0.1 to $4.0, E_{s}$ is the modulus of the solid parent material, and $\rho_{f}$ is the relative density of the foam. Relative density of foams is defined as the density of the foam divided by the density of the parent material from which it is foamed, expressed as a percentage. By substitution of Eq. (3) into Eq. (2), the assumption that only the face sheets carry in-plane loads is shown to be valid for the lowdensity foams of interest in most sandwich structure applications.

\section{B. Method Validation}

To verify the accuracy of this technique, a thin square 5052-H32 aluminum plate was placed in the 1018 steel frame and subjected to a quasi-statically increasing $\left(1^{\circ} \mathrm{C} / \mathrm{min}\right)$ uniform temperature field. The plate thickness is $3.175 \mathrm{~mm}(0.125$ in.) with a $216 \times 216 \mathrm{~mm}$ $(8.5 \times 8.5$ in. $)$ test section. Verification of the method was obtained by comparing the measured thermal buckling load with that predicted by classical thin plate theory,

$$
\Delta T_{\text {cr }}=\left(4 \pi^{2} / 9\right)\left\{1 /\left[\left(\alpha_{\text {frame }}-\alpha_{\text {panel }}\right)(1+v)\right]\right\}(t / a)^{2}
$$

in which $\Delta T_{\mathrm{cr}}$ is the temperature at bifurcation and $t / a$ is the ratio of plate thickness to edge length.

Figure 7 shows the strain vs temperature for one of the plate specimens measured by back-to-back strain-gauge pairs and the 

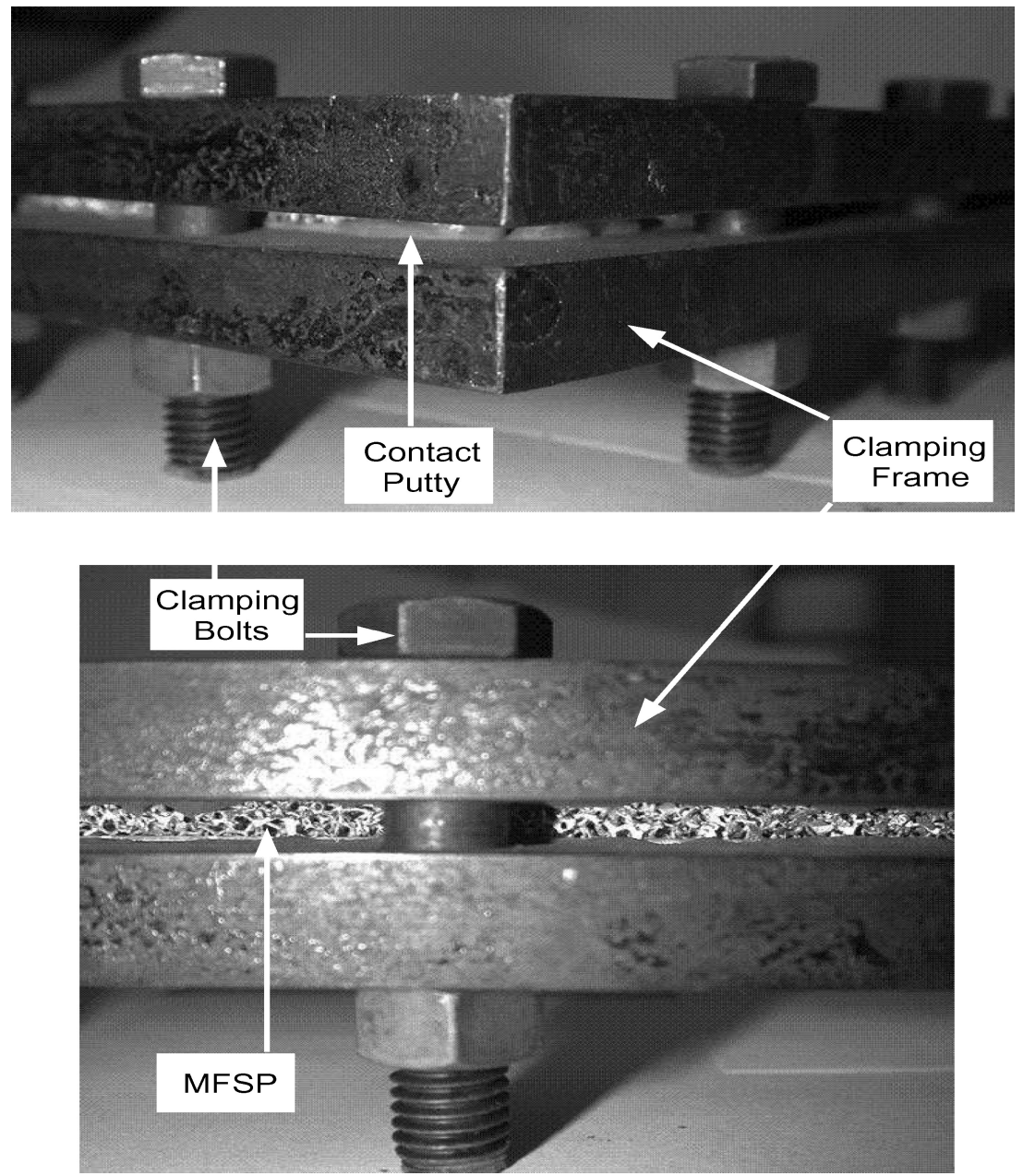

Fig. 6 Details of steel load frame.

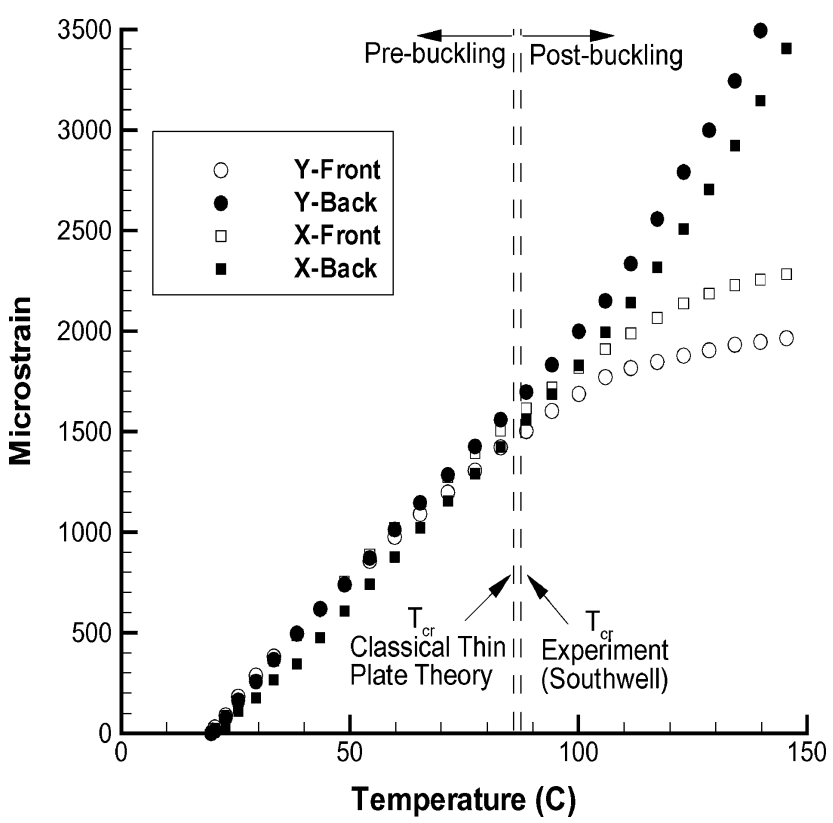

Fig. 7 Response of thin plate to thermal loading that demonstrates validity of the experimental technique.
Table 1 Critical temperatures of thin monolithic 5052-H32 aluminum plates

\begin{tabular}{lcc}
\hline \hline Plate & $\Delta T_{\text {cr }},{ }^{\circ} \mathrm{C}$ & Difference, $\%$ \\
\hline Theory & 62 & $-\overline{1.6}$ \\
1 & 63 & 4.8 \\
2 & 59 & \\
\hline \hline
\end{tabular}

comparison of the measured bifurcation point with that predicted by Eq. (4). The precise bifurcation point was determined with a Southwell plot $^{17}$ and strain decomposition, as are used and explained in the following section for MFSPs. The data indicate agreement between theory and experiment to within 5\%, which verifies that this experimental technique induces in-plane loading and bifurcation in the fashion predicted by Eq. (4). A second monolithic aluminum plate was tested to establish repeatability in the verification of this technique. The experimentally measured critical buckling temperatures of the two plates are listed in Table 1, along with the theoretical prediction from Eq. (4).

\section{MFSP Specimens and Instrumentation}

The aluminum foam sandwich panels analyzed in the present work are shown in Fig. 8. The panels measure $254 \times 254 \mathrm{~mm}$ $(10 \times 10$ in.). The core has a thickness of $6.35 \mathrm{~mm}(0.25 \mathrm{in}$.). The foam is made from 6101-T6 aluminum and has eight pores per 


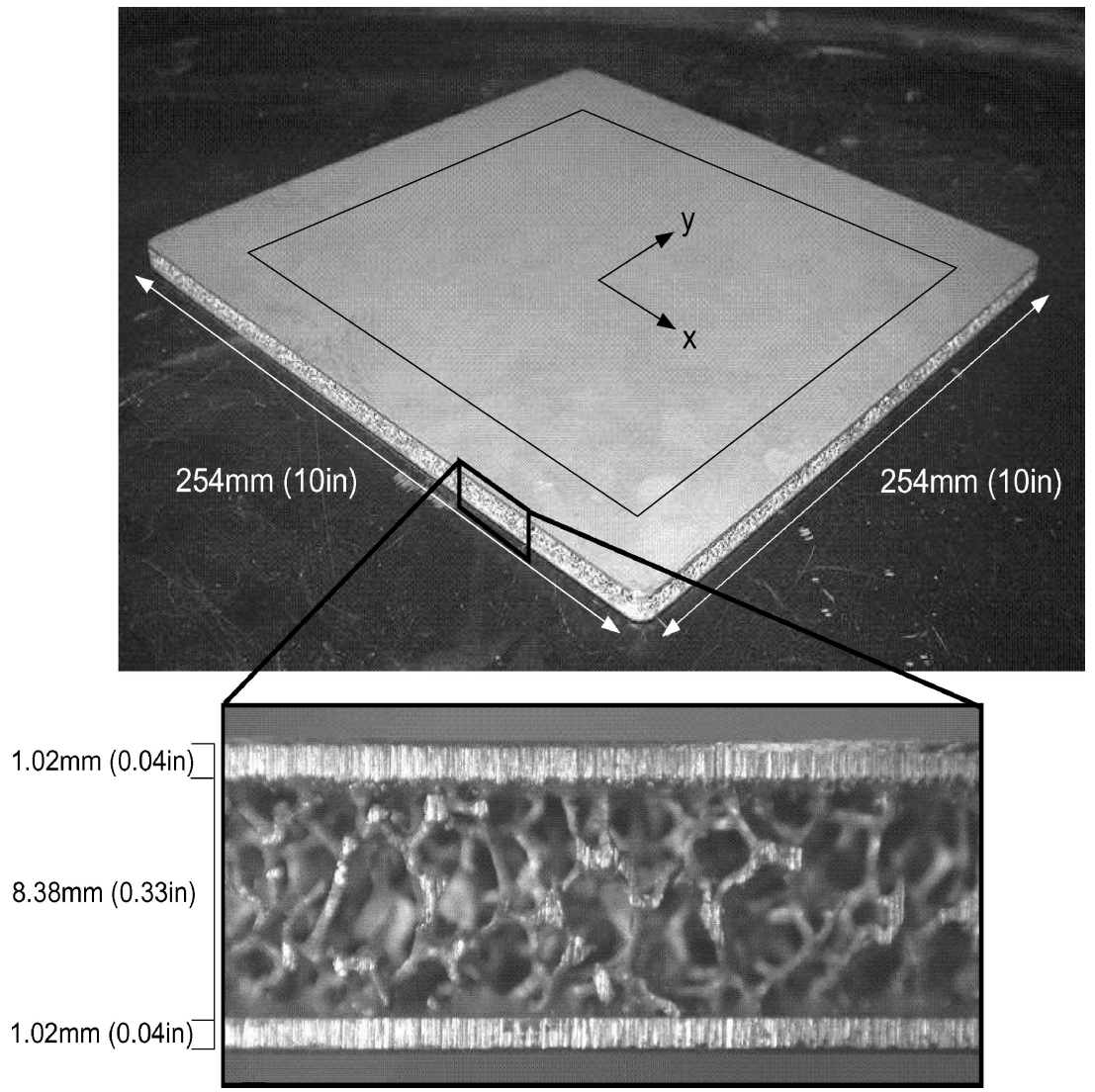

Fig. 8 MFSPs used to obtain results of present work.

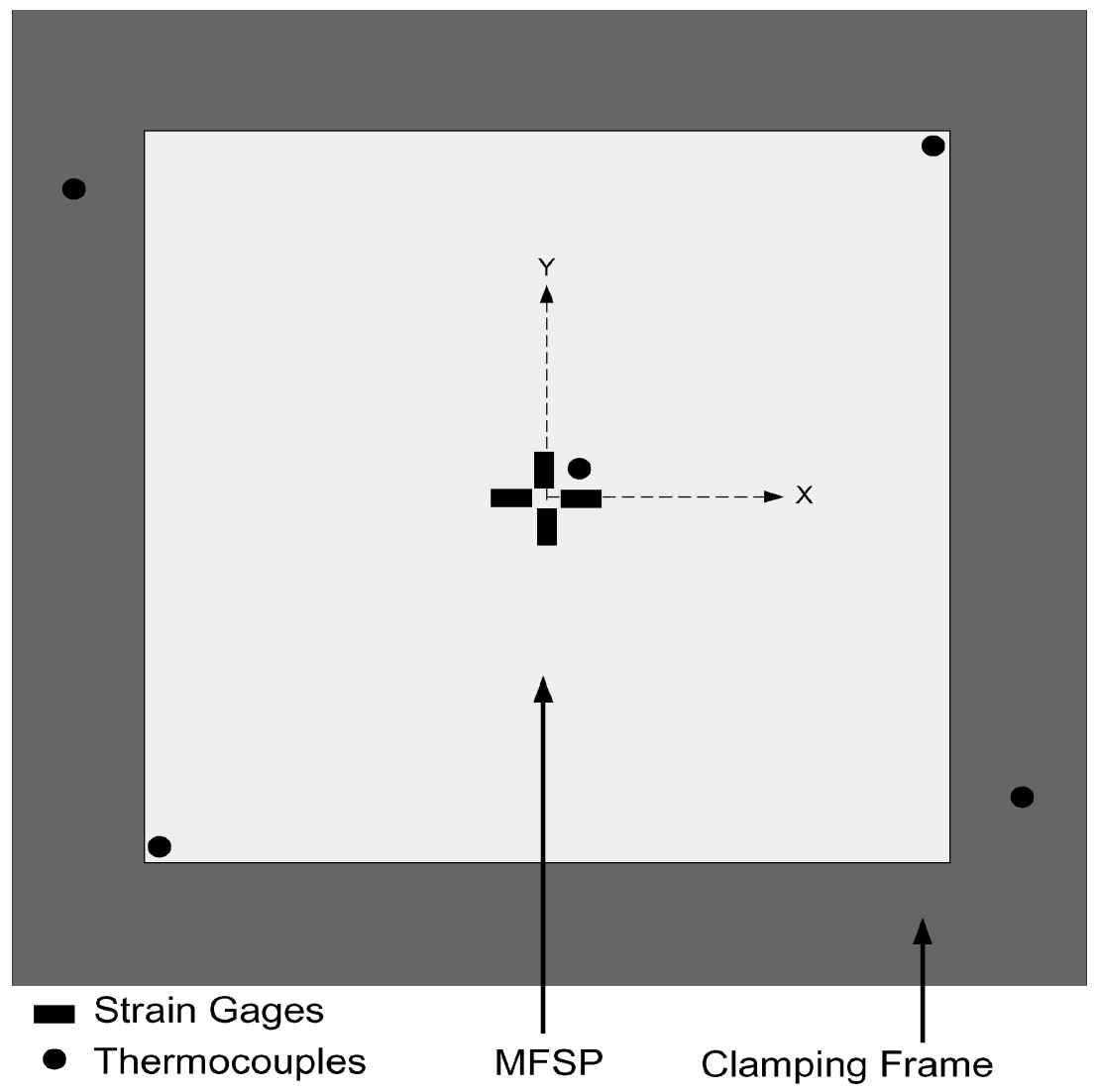

Fig. 9 Location of strain gauges and thermocouples on instrumented panel-frame setup; instrument locations are symmetric about the $x-y$ plane. 
centimeter (20 pores per inch) and a relative density of $\rho_{f}=8 \%$. The core is brazed to 6061-T6 aluminum face sheets, which have a thickness of $1 \mathrm{~mm}$ (0.04 in.). The panels were manufactured by ERG Materials and Aerospace Corp. (Oakland, California). Four identical specimens were subjected to uniform thermal loading and unloading in the steel frame.

An instrumented panel-frame setup is shown in Fig. 9. Each of the specimens was instrumented with five distributed type-K Nextelinsulated thermocouples (XC-20-K-72, Omega Engineering) with electronic ice point reference junctions. The locations of the five thermocouples varied among the tests and were chosen from the locations shown in Fig. 9. The purpose of the range of locations was to ensure that the panel-frame assembly experienced no thermal gradients, neither in the plane of the panel nor through the thickness of the assembly. Each combination of thermocouple locations confirmed a uniform temperature field throughout the entire assembly over the duration of the experiment, as can be seen by the thermal loading curve in Fig. 10.

Two of the specimens were instrumented with high-temperature, fully encapsulated Karma-based alloy strain gauges (WK-062AP13-350, Vishay Micromeasurements), arranged in back-to-back pairs located in the center of each face sheet of the panel, parallel and perpendicular to the boundary of the test section. Multiple coaxial strain gauges were used for redundancy in the measurement system.

The other two specimens were instrumented for shadow moire interferometry, ${ }^{18}$ measuring the out-of-plane displacement field as the panel buckled and deformed into the postbuckling regime. In the shadow moire method, as shown in Fig. 11, a specimen is put in contact with a ronchi ruling, which is a glass substrate marked with parallel black lines evenly spaced at a pitch $p$. A white light source shines through the ruling onto the specimen at a nonzero angle $\beta$ with respect to the normal axis of the specimen. The light reflects off of the surface of the specimen, passes back through the ruling, and produces an interference pattern that is captured by a digital camera also oriented at a nonzero angle $\gamma$. The interference pattern is a contour representation of out-of-plane displacements. Each fringe represents a differential displacement magnitude $\Delta w$ such that

$$
\Delta w=p /(\cos \beta+\cos \gamma)
$$

In the present experiments, the light source and camera are oriented at angles of $\beta=8.5 \mathrm{deg}$ and $\gamma=9.7 \mathrm{deg}$, respectively, from the normal axis of the panel. With a pitch of 12 lines/mm (300 lines/in.)

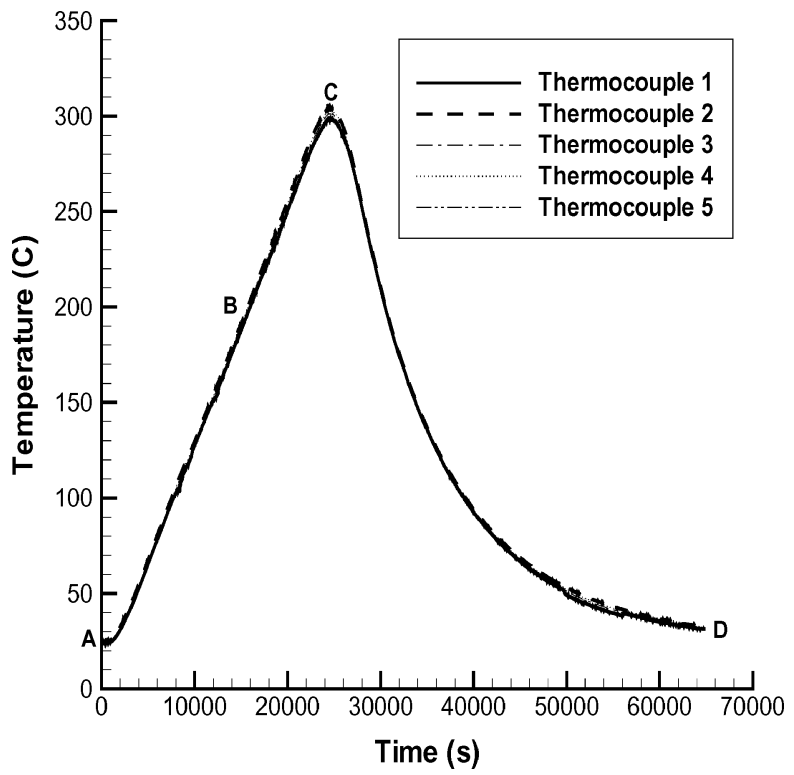

Fig. 10 Five simultaneous thermocouple readings that demonstrate uniformity of applied temperature in experiments.

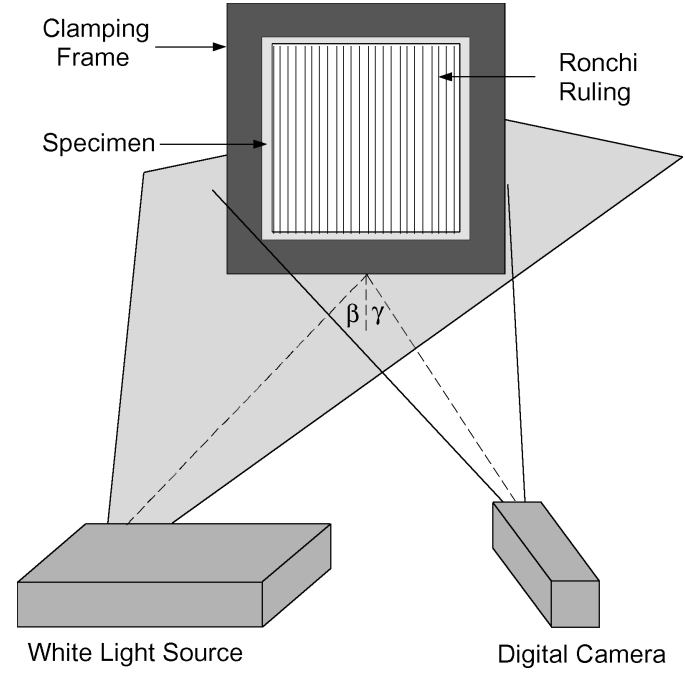

Fig. 11 Setup for measuring buckling modes with shadow moire interferometry.

on the glass substrate, the increment of out-of-plane deflection represented by each fringe is $\Delta w=0.264 \mathrm{~mm}$ (0.0104 in.).

With an average temperature defined by the thermocouple measurements, the local bending and membrane strains measured by the strain gauges and the full out-of-plane displacement field measured by the moire fringe patterns provide a temperature-based history of the thermally induced prebuckling, buckling, and postbuckling response of MFSPs.

\section{Thermal Loading}

The panel-frame assembly was subjected to uniform thermal loading in an oven. The temperature-time history is a simple loadunload quasi-static cycle with a temperature rise rate of $1{ }^{\circ} \mathrm{C} / \mathrm{min}$, a peak temperature of $300^{\circ} \mathrm{C}$, and an uncontrolled free convection cool-down to room temperature. The maximum temperature of the oven dictated the peak temperature of the experimental loading cycle. This loading cycle is shown in Fig. 10, which shows the temperature measurement of five thermocouples distributed across both sides of the panel-frame assembly. Thermal gradients are shown to be minimal or nonexistent throughout the duration of the experiment.

\section{Results and Discussion}

\section{A. In-Plane Response}

The response of MFSPs to uniform thermal loading and unloading is shown in Fig. 12. As the temperature rises, equibiaxial tensile strains develop equivalently on each side of the panel. From points A to B in Fig. 12, the panel is in the prebuckled state. The tensile aggregate strain state is accompanied by a compressive aggregate stress state. This is possible because a nonzero temperature change, $\Delta T \neq 0$, is introduced into the thermoelastic constitutive relations,

$$
\sigma_{i j}=\lambda \delta_{i j} \epsilon_{k k}+2 G \epsilon_{i j}-(3 \lambda+2 G) \delta_{i j} \alpha \Delta T
$$

The tensile aggregate strains have a positive thermal strain component and a negative mechanical strain component, due to the constricting frame, that result in compressive stresses in the panel. The magnitudes and signs of the thermal and mechanical strain components during the load-unload thermal cycle will be discussed in further detail at the end of this section.

At point $\mathrm{B}$, the panel buckles and the strain states on opposite sides of the panel diverge and continue diverging until the peak temperature at point $\mathrm{C}$. The strain response at and around the point of buckling is shown in Fig. 13. The panel buckles symmetrically 


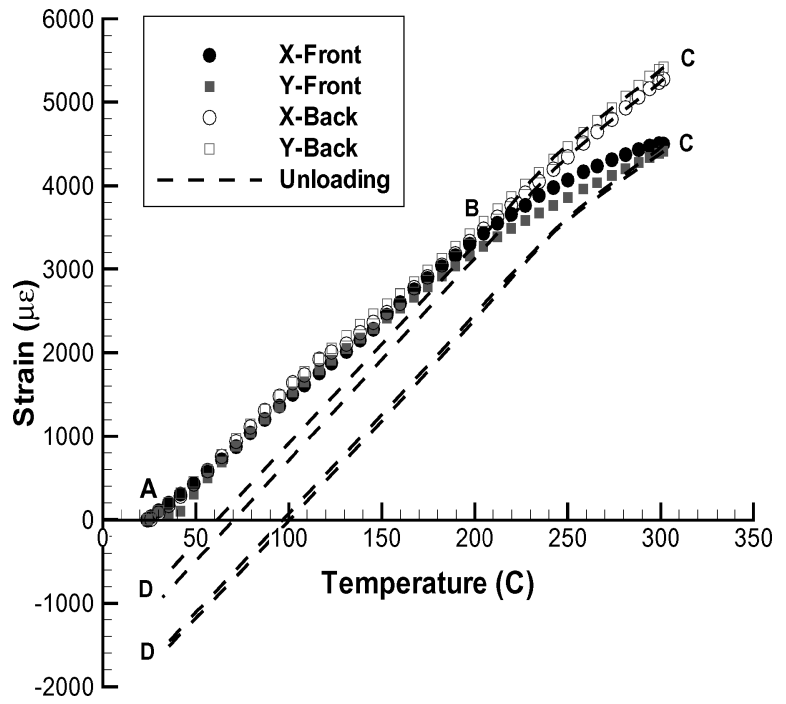

Fig. 12 Response of MFSPs to thermal loading and unloading.

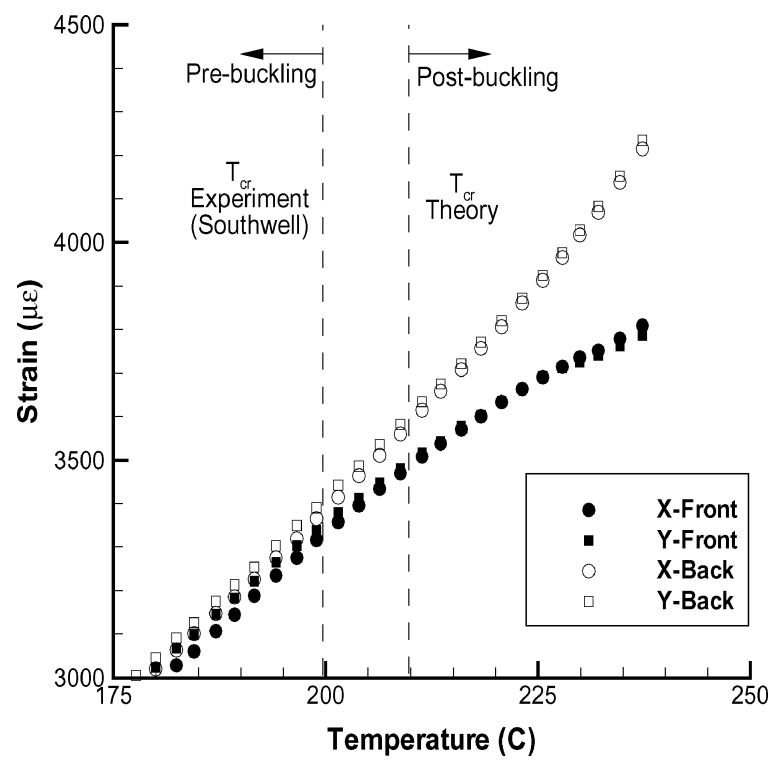

Fig. 13 Response of MFSPs to thermal loading at the point of thermal buckling.

in both the $x$ and $y$ directions, and the shape can be represented by

$$
\begin{gathered}
w(x, y)=\sin \left(\frac{\pi x}{a}\right) \sin \left(\frac{\pi y}{a}\right) \sum_{m=1}^{\infty} \sum_{n=1}^{\infty} A_{m n} \\
\times \sin \left(\frac{m \pi x}{a}\right) \sin \left(\frac{n \pi y}{a}\right)
\end{gathered}
$$

in which $w(x, y)$ is the magnitude of out-of-plane deflection. Equation (7) is the series of mode shapes used in the Rayleigh-Ritz analysis that will be formulated in a later subsection of this paper. This analysis considers a plate with clamped boundary conditions, as satisfied by Eq. (7).

Beyond buckling, between points $\mathrm{B}$ and $\mathrm{C}$, it is clear from Fig. 12 that the aggregate strain on the front side of the panel becomes increasingly compressive relative to the aggregate strain on the back side of the panel. This behavior continues until the peak temperature at point $\mathrm{C}$. From points $\mathrm{C}$ to $\mathrm{D}$, the temperature decreases through free convection, releasing all elastic thermal and mechanical strain components. Once unloaded, the accumulated plastic strain in the panel is shown at point D. A detailed analysis of the thermoelasticplastic strain components is given later in this section.
A critical design criterion for in-plane loads in TPSs, such as actively cooled MFSPs, is the buckling point. Although a buckling point is identifiable in Fig. 13, a more accurate numerical value may be obtained by transforming the strain-temperature measurements into two different forms. The first is a decomposition of the strain into bending and membrane modes as is shown in Fig. 14. From Fig. 14, it is clear that the panel is in a state of pure membrane deformation until a distinct onset of nonzero bending strain, which indicates buckling within the panel.

The second useful transformation of data is a temperature-based Southwell plot for plate buckling (see Ref. 17). A typical Southwell plot, for the mechanical buckling of columns, is obtained by considering

$$
w=P_{\mathrm{cr}}(w / P)-w_{0}
$$

and plotting $w / P$ vs $w$. Here, $w$ is the lateral deflection of the midpoint of the column, $w_{0}$ is the amplitude of initial curvature in the imperfect column, and $P$ is the end load. The inverse slope of the Southwell plot is the buckling load of the column. The analog to this procedure for thermal buckling in panels is represented by

$$
\epsilon_{b}=\left(\Delta T_{\mathrm{cr}} / \Delta T\right) \epsilon_{b}-w_{0, x x}
$$

and the inverse slope of a plot of $\epsilon_{b} / \Delta T$ vs $\epsilon_{b}$ yields the buckling temperature $\Delta T_{\text {cr }}$ of the panel. This, is shown in Fig. 15.

Both the strain decomposition technique and the Southwell method are used to identify the buckling point of the two panels instrumented with strain gauges. The buckling temperatures $\Delta T_{\text {cr }}$ of the four MFSP specimens are shown in Table 2 with a comparison to a theoretical prediction. The results show repeatability and close correlation to theory. In Sec. III.B, the results from experiments with MFSPs instrumented for moire interferometry are explained in detail. In Sec. III.C, the theoretical development is discussed.

The temperature history of the thermal and mechanical elastic and plastic strain components, throughout the duration of the loading cycle, are shown in Fig. 16. The aggregate strain state is decomposed

Table 2 Critical temperatures of aluminum foam sandwich panels

\begin{tabular}{lccl}
\hline \hline Panel & $\Delta T_{\mathrm{cr}},{ }^{\circ} \mathrm{C}$ & Difference, $\%$ & Measurement method \\
\hline Theory & 167 & - & - \\
1 & 188 & 12.6 & Strain gauges \\
2 & 175 & 4.8 & Strain gauges \\
3 & 170 & 1.8 & Moire interferometry \\
4 & 176 & 5.4 & Moire interferometry \\
\hline \hline
\end{tabular}

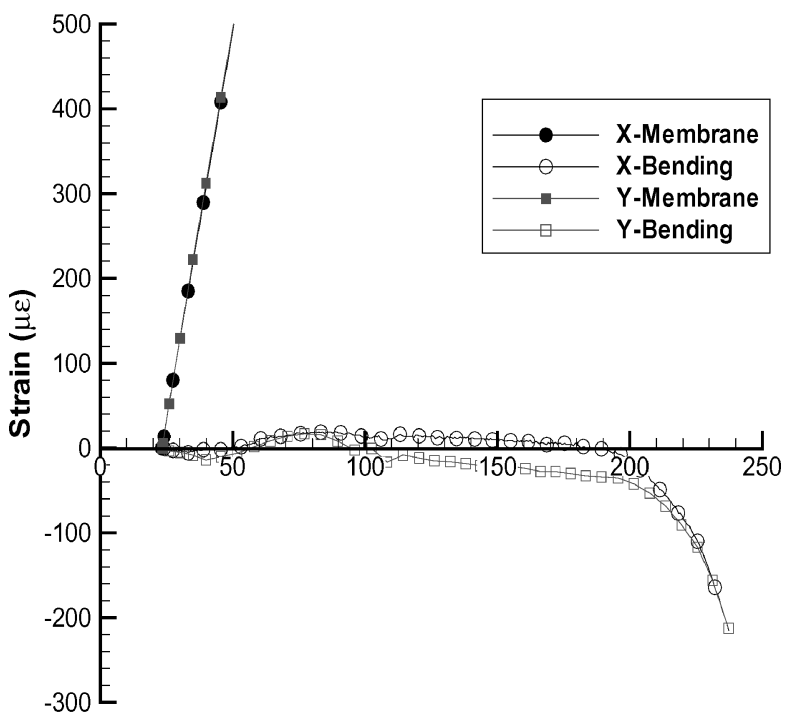

Temperature $(C)$

Fig. 14 Strain history of the MFSP decomposed into bending and membrane strain. 


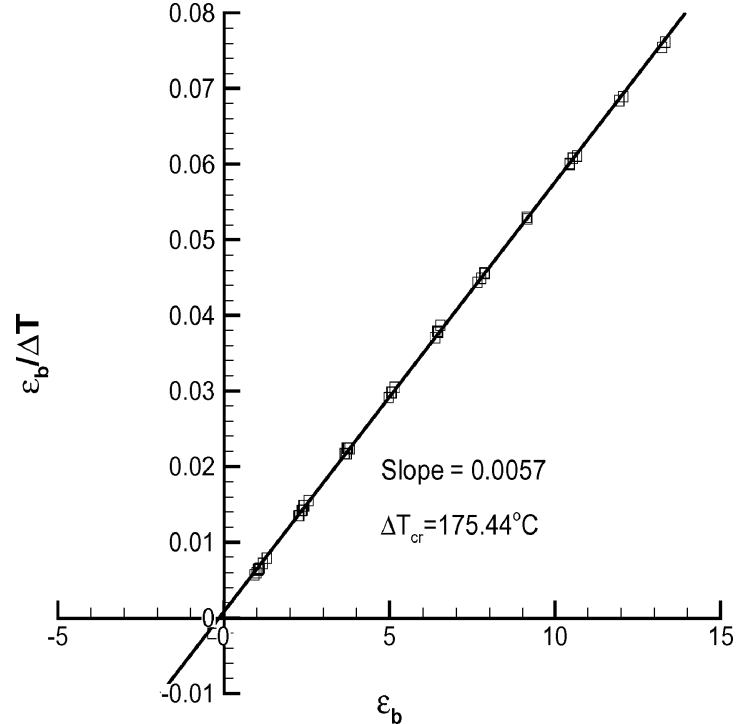

Fig. 15 Southwell plot that determines the buckling temperature of the MFSPs.

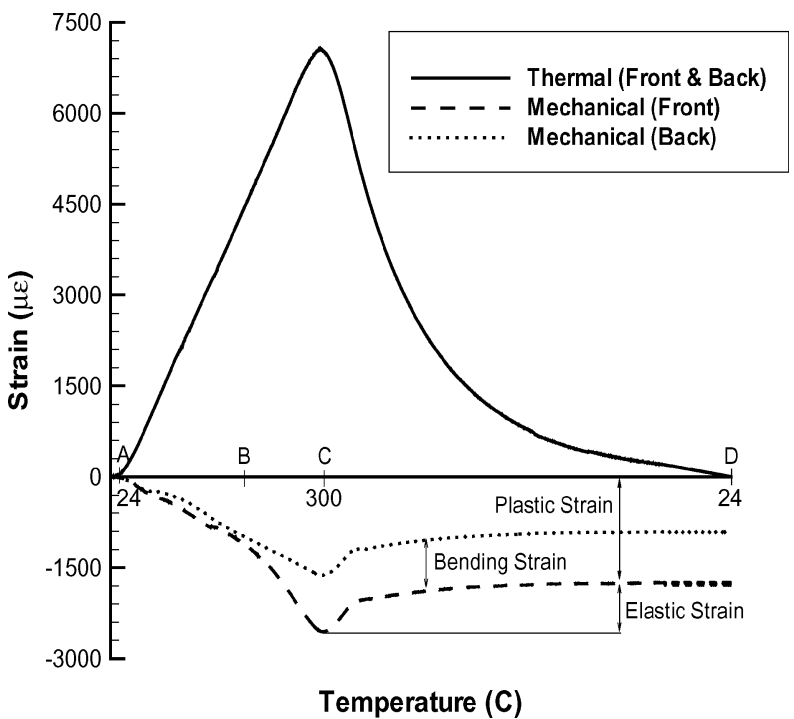

Fig. 16 Thermal and mechanical strain throughout the loading cycle.

into its thermal and mechanical (elastic and plastic) components. This decomposition is possible because $\alpha(T)$ and $\Delta T(t)$ are known for the MFSPs.

Throughout the duration of the experiment, points $\mathrm{A}-\mathrm{D}$, the thermal strain is positive in the panel, as is dictated when $\Delta T \geq 0$ in a material for which $\alpha(T)>0$. The magnitude of thermal strain dominates the aggregate strain state throughout the loading cycle and most of the unloading cycle of the experiment. From point A to point $B$ to point $C$, the mechanical strain state follows qualitatively similar behavior as the aggregate strain analysis described in detail earlier and in Figs. 12 and 13. The mechanical strains are similar on both sides of the panel until buckling, at which point the strains diverge until the peak temperature is reached at point $\mathrm{C}$.

From point $\mathrm{C}$ to point $\mathrm{D}$, Fig. 16 illustrates elastoplastic behavior that could not be revealed by the aggregate strain analysis. Thermal unloading initially produces a decrease in mechanical strain (elastic strain) of approximately $22 \%$ of the peak mechanical strain on each side of the panel. After this initial relaxation, the mechanical strain reaches a plastic strain plateau for the remainder of the thermal unloading. Although elastic mechanical strain is released, the bending strain, the difference between the mechanical strains on each side of the panel, remains constant for the entire unloading cycle. This is readily apparent in Fig. 16. The release of elastic strain, then, is exclusively membrane strain, attributed to the loss of in-plane loading from the constricting frame. The curvature that was present in the panel at the peak temperature (point $C$ ) is wholly present when the panel is completely unloaded (point $\mathrm{D}$ ). This behavior will be visualized with shadow moire interferometry in the next section.

\section{B. Out-of-Plane Response}

An analysis by shadow moire interferometry is used to identify the critical temperatures, the buckling mode shapes, and the postbuckling response of the MFSPs. In situ images of the MFSPs under thermal loading were captured throughout the response experiment. Figure 17 shows the moire fringe patterns captured at each of the lettered response points in Fig. 12. At point A, there is a slight fringe pattern that indicates the level of imperfection in the measurement system: initial panel curvature, misalignment of the ronchi ruling, or curvature in the ruling. This image serves as a reference state for the analysis. The image at point $\mathrm{A}$ remains unchanged until point $\mathrm{B}$ because there is no appreciable relative out-of-plane deformation in the prebuckling regime.

At point B, the first sign of thermomechanical out-of-plane curvature appears as a black spot at the center of the panel, which is indicated by the white arrow in Fig. 17. This occurs at the same temperature at which the strain-gauge measurements begin to diverge in Figs. 12 and 13. The appearance of the black spot in the center of the panel indicates the first critical temperature of the MFSPs. From point $\mathrm{B}$ to point $\mathrm{C}$, the fringe pattern evolves as the panel continues to deflect out of the plane of loading with a new fringe appearing and growing from the center of the panel each time the center of the panel deflects $\Delta w=0.264 \mathrm{~mm}$ (0.0104 in.).

At point $\mathrm{C}$, the panel is fully loaded $\left(T=300^{\circ} \mathrm{C}\right)$ and a clear set of fringes provide a precise measurement of the out-of-plane deflection over the entire surface of the panel. After point $C$, the panel is unloaded and all elastic deformation is released.

At point $\mathrm{D}$, when the panel is fully unloaded, the fringe pattern has not changed appreciably from point $C$. This indicates that only a small amount of the out-of-plane deflection is elastic for the geometry and thermal loading used in the present investigation. The images between points $\mathrm{C}$ and $\mathrm{D}$ confirm the findings of the elastoplastic strain analysis of the preceding section. Thermal unloading of these MFSPs released membrane strain, whereas the bending strain proved to be plastic.

It would be informative to obtain measurements of MFSPs further into the postbuckling regime than those presented in Fig. 17. However, the peak temperature of the oven used in the present experiments prohibits the investigation of the postbuckling response of MFSPs at higher temperatures. For related experiments involving higher temperatures, the reader is referred to Ref. 19. To further investigate the postbuckling response of MFSPs without requiring higher test temperatures, an imperfection was introduced into a fifth MFSP specimen to induce relatively large out-of-plane deflections early in the thermal loading history and to allow imaging of the postbuckling mode shapes across a larger portion of the postbuckling regime.

To introduce the imperfection into the MFSP, the clamping bolts on the frame were overtightened such that the edges of the entire perimeter of the panel were forced to curve toward each other, into the core, creating an initial curvature in each face of the panel. The imperfect panel was then subjected to the same thermal loading that the perfect panels experienced, and images were captured of the moire fringe patterns. Figure 18 shows the postbuckling mode shapes of the MFSP as the temperature rises above the critical temperature of the imperfect panel.

The images of the fringe patterns are quantified into data that highlight the thermal postbuckling behavior of MFSPs. Figure 19 shows the magnitude of out-of-plane deflection of the center point of the panel vs normalized temperature, $\Delta T / \Delta T_{\mathrm{cr}}$. The onset of buckling is followed by a rapid increase in out-of-plane deflection. This initial buckling behavior relaxes into a steady postbuckling response. 

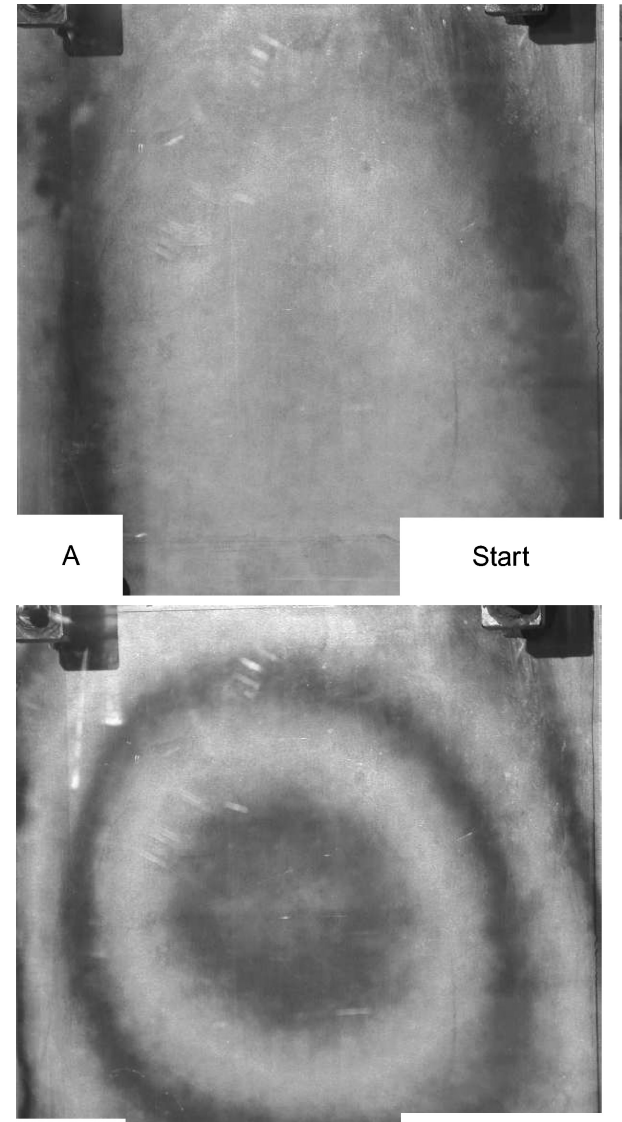

C

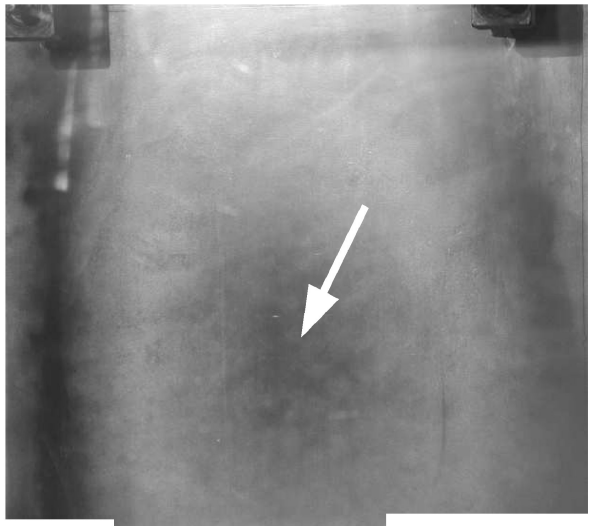

$\mathrm{Tcr}$

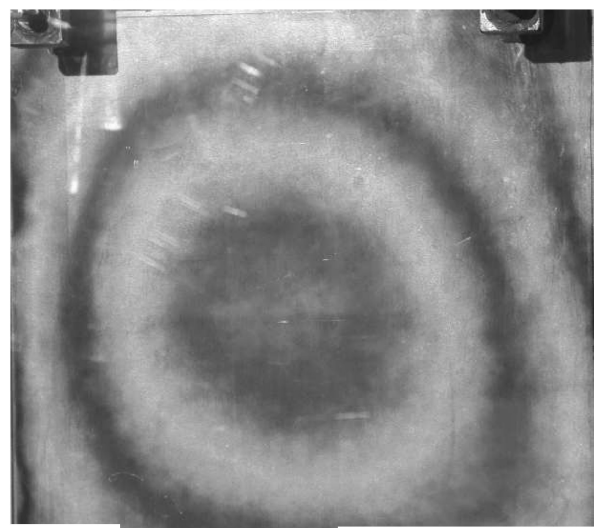

Unloaded

Fig. 17 Digital images of moire fringes that show full-field buckling modes on the surface of an MFSP; each fringe represents $\Delta w=0.264$ mm (0.0104 in.).
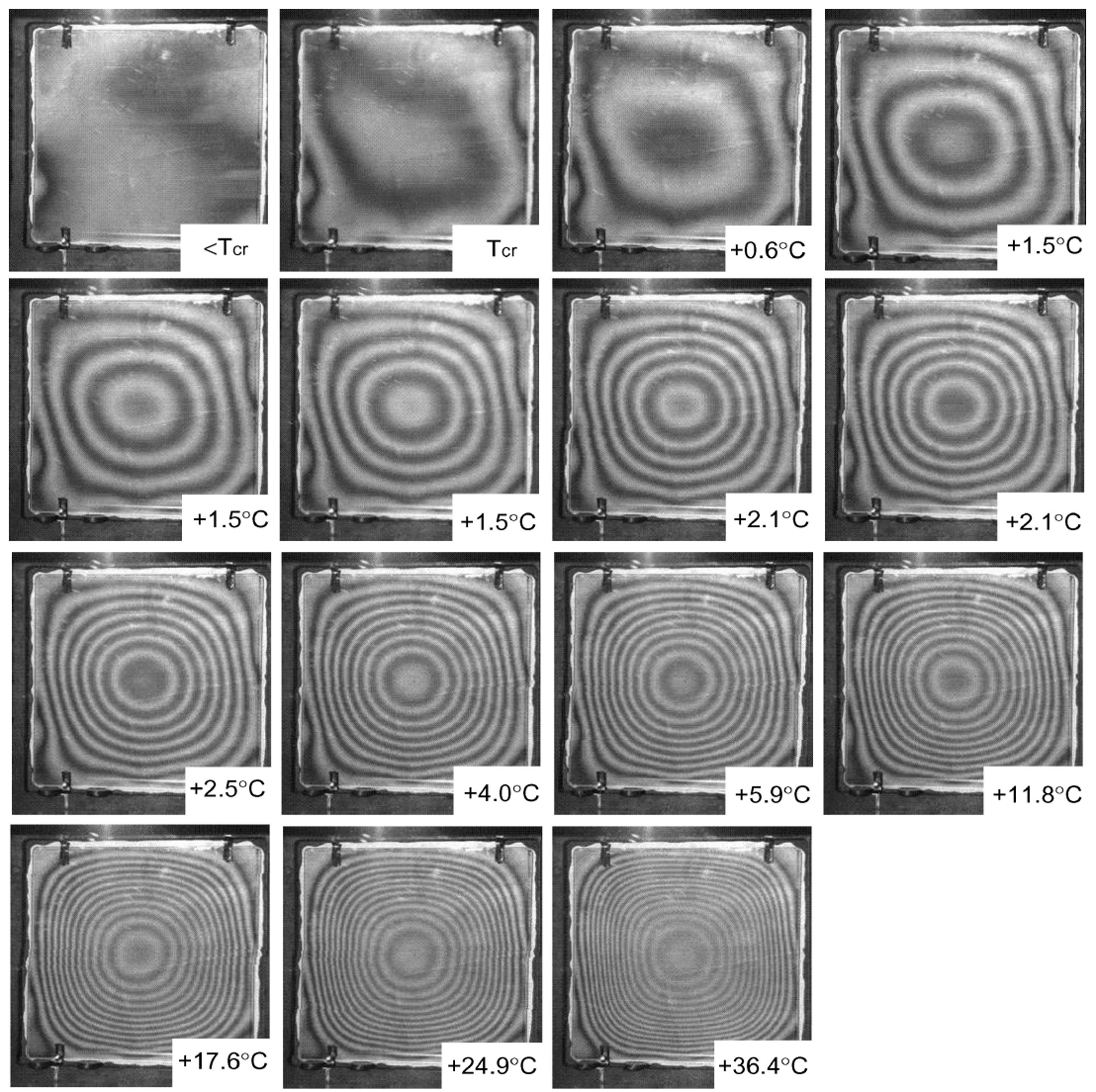

Fig. 18 Digital images of moire fringes that show full-field buckling modes on the surface of the initially curved MFSP; each fringe represents $\Delta w=0.264 \mathrm{~mm}(0.0104$ in. $)$. 


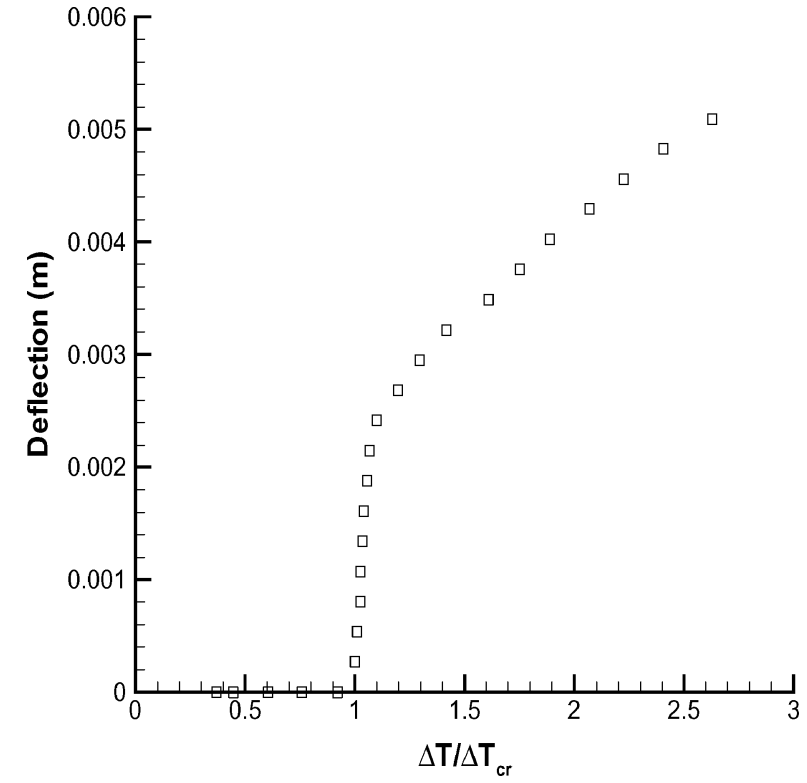

Fig. 19 Amplitude of the buckled mode shape throughout the thermal response of the MFSP.

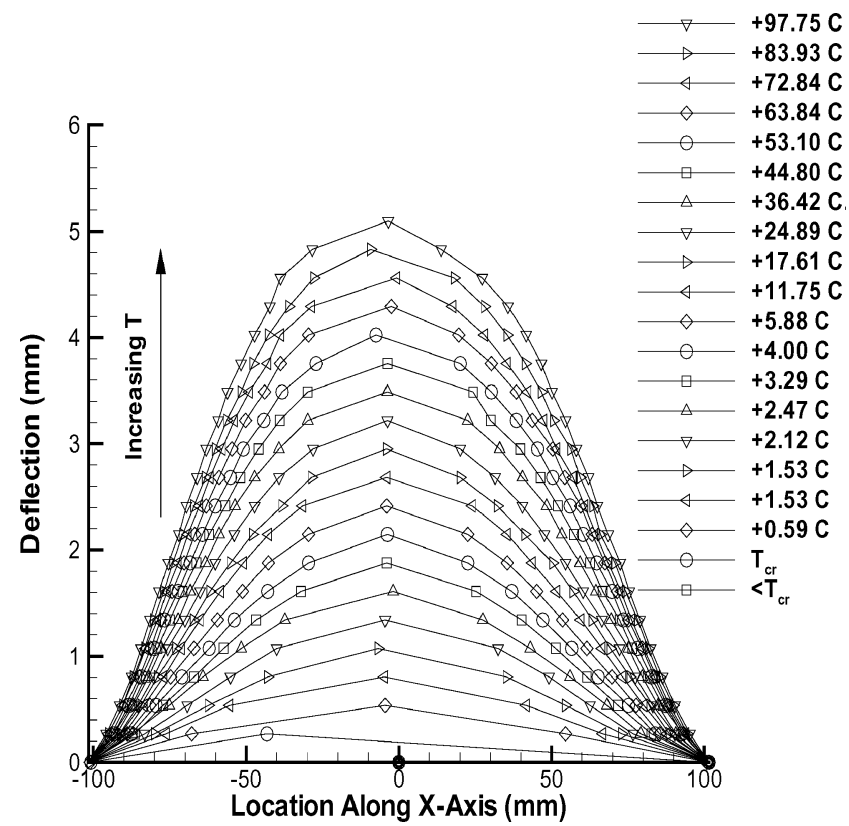

Fig. 20 Response history of line lying along $x$ axis on surface of the MFSP.

The evolution of the mode shapes throughout postbuckling is further illustrated by considering the deflection of a line on the surface of the panel as the temperature increases beyond bifurcation. Figure 20 shows the out-of-plane deflection at postbuckling temperatures of a line lying along the $x$ axis of the specimen, as quantified through the moire fringe patterns. Because of symmetry, the response of the material along the $x$ axis is equivalent to that of material along the $y$ axis. It is clear from Fig. 20 that the center portion, that is, the middle $40 \mathrm{~mm}$, of the panel remains relatively flat throughout the postbuckling response, whereas deformation accumulates in the lobes of the curvature in the form of an increasing slope.

This finding adds further insight to the strain analysis discussed in the preceding section. The strain gauges, as shown in Fig. 9, are located at $x=0 \mathrm{~mm}$ in Fig. 20. The bending strain measured by the gauges at the center of the panel, shown in Fig. 14, is then a representation of curvature at $x=0$. At the onset of buckling, the maximum bending strain is shown to be at $x=0$. As the mode shapes evolve, however, Fig. 20 shows that the maximum bending strain and stress is no longer in the center of the panel. This can be seen in Fig. 18 as well. The density of the contours shifts from the center of the MFSP during initial buckling toward the boundary of the panel in the postbuckling regime.

\section{Comparison with Theory}

The energy formulation for the thermal loading of a sandwich panel, as developed in Refs. 8 and 9, is presented and adapted to the problem at hand. The formulation considers a rectangular sandwich panel under thermal loading due to a static uniform temperature change. The sandwich panel is considered to be a shear-deformable plate, such that there is no account of the cross-sectional kinematics of a sandwich panel, specifically. The rotation of a cross-sectional normal is represented by a single variable, akin to a ReissnerMindlin formulation. The geometry and material properties of the panel core determine the constitutive properties of the plate under shear loading, whereas the geometry and material properties of the face sheets determine the constitutive properties of the plate under in-plane loads and bending moments. By the use of standard notation for laminated plate theory, ${ }^{20}$ the constitutive relations for the sandwich panel are taken to be

$$
\begin{aligned}
& {\left[\begin{array}{c}
N_{x} \\
N_{y} \\
N_{x y}
\end{array}\right]=\left[\begin{array}{ccc}
\bar{A}_{11} & \bar{A}_{12} & 0 \\
\bar{A}_{21} & \bar{A}_{22} & 0 \\
0 & 0 & \bar{A}_{66}
\end{array}\right]\left[\begin{array}{c}
\frac{\partial u}{\partial x} \\
\frac{\partial v}{\partial y} \\
\frac{\partial u}{\partial y}+\frac{\partial v}{\partial x}
\end{array}\right]-\left[\begin{array}{c}
N_{x}^{T} \\
N_{y}^{T} \\
N_{x y}^{T}
\end{array}\right]} \\
& {\left[\begin{array}{c}
M_{x} \\
M_{y} \\
M_{x y}
\end{array}\right]=\left[\begin{array}{ccc}
\bar{D}_{11} & \bar{D}_{12} & 0 \\
\bar{D}_{21} & \bar{D}_{22} & 0 \\
0 & 0 & \bar{D}_{66}
\end{array}\right]} \\
& \times\left[\begin{array}{c}
-\frac{\partial}{\partial x}\left(\frac{\partial w}{\partial x}-\gamma_{x z}\right) \\
-\frac{\partial}{\partial y}\left(\frac{\partial w}{\partial y}-\gamma_{y z}\right) \\
-\frac{\partial}{\partial y}\left(\frac{\partial w}{\partial x}-\gamma_{x z}\right)-\frac{\partial}{\partial x}\left(\frac{\partial w}{\partial y}-\gamma_{y z}\right)
\end{array}\right]-\left[\begin{array}{c}
M_{x}^{T} \\
M_{y}^{T} \\
M_{x y}^{T}
\end{array}\right]
\end{aligned}
$$

in which

$$
\begin{aligned}
& {\left[\begin{array}{ccc}
N_{x}^{T} & , & M_{x}^{T} \\
N_{y}^{T} & , & M_{y}^{T} \\
N_{x y}^{T} & , & M_{x y}^{T}
\end{array}\right]=\sum_{i=1}^{2}\left[t_{s} T_{i},(-1)^{i} \frac{t_{s} h}{2} T_{i}\right]} \\
& \times\left[\begin{array}{ccc}
\frac{E}{1-v^{2}} & \frac{E v}{1-v^{2}} & 0 \\
\frac{E v}{1-v^{2}} & \frac{E}{1-v^{2}} & 0 \\
0 & 0 & G_{x y}
\end{array}\right]_{i}\left[\begin{array}{c}
\alpha_{x} \\
\alpha_{y} \\
\alpha_{x y}
\end{array}\right]_{i} \\
& {\left[\begin{array}{l}
Q_{x} \\
Q_{y}
\end{array}\right]=\left[\begin{array}{cc}
D_{Q_{x}} & 0 \\
0 & D_{Q_{y}}
\end{array}\right]\left[\begin{array}{l}
\gamma_{x z} \\
\gamma_{y z}
\end{array}\right]}
\end{aligned}
$$

The boundary conditions are such that in-plane displacements vanish along the boundary of the panel, and, in the case considered in the present work, all edges are clamped. Accordingly, the outof-plane deflection $w(x, y)$ and the rotation of the cross-sectional 
normals $\gamma_{x z}$ and $\gamma_{y z}$ must vanish along the boundary of the panel. Appropriate shape functions are

$$
\begin{aligned}
& w(x, y)=\sin \left(\frac{\pi x}{a}\right) \sin \left(\frac{\pi y}{b}\right) \sum_{m=1}^{\infty} \sum_{n=1}^{\infty} A_{m n} \\
& \times \sin \left(\frac{m \pi x}{a}\right) \sin \left(\frac{n \pi y}{b}\right) \\
& \gamma_{x z}(x, y)=\cos \left(\frac{\pi x}{a}\right) \sin \left(\frac{\pi y}{b}\right) \sum_{m=1}^{\infty} \sum_{n=1}^{\infty} B_{m n} \\
& \times \sin \left(\frac{m \pi x}{a}\right) \sin \left(\frac{n \pi y}{b}\right)+\sin \left(\frac{\pi x}{a}\right) \sin \left(\frac{\pi y}{b}\right) \\
& \times \sum_{m=1}^{\infty} \sum_{n=1}^{\infty} m B_{m n} \cos \left(\frac{m \pi x}{a}\right) \sin \left(\frac{n \pi y}{b}\right) \\
& \gamma_{y z}(x, y)=\sin \left(\frac{\pi x}{a}\right) \cos \left(\frac{\pi y}{b}\right) \sum_{m=1}^{\infty} \sum_{n=1}^{\infty} C_{m n} \\
& \times \sum_{m=1}^{\infty} \sum_{n=1}^{\infty} n C_{m n} \sin \left(\frac{m \pi x}{a}\right) \cos \left(\frac{n \pi y}{b}\right) \\
& \times \sin \left(\frac{m \pi x}{a}\right) \sin \left(\frac{n \pi y}{b}\right)+\sin \left(\frac{\pi x}{a}\right) \sin \left(\frac{\pi y}{b}\right)
\end{aligned}
$$

The potential energy of the heated panel is minimized with respect to each of the degrees of freedom, such that

$$
\frac{\partial \Pi}{\partial A_{m n}}=\frac{\partial \Pi}{\partial B_{m n}}=\frac{\partial \Pi}{\partial C_{m n}}=0
$$

produces three equations for each $m n k l$, relating $A_{m n}, B_{m n}$, and $C_{m n}$,

$$
\begin{gathered}
\sum_{k}^{\infty} \sum_{l}^{\infty}\left\{\left[a_{m n k l}^{11}+\eta \frac{D^{\star}}{a b}\left(\frac{\pi}{a}\right)^{2} k_{x y} \delta_{m n k l}\right] A_{k l}\right. \\
\left.+a_{m n k l}^{12} B_{k l}+a_{m n k l}^{13} C_{k l}\right\}=0
\end{gathered}
$$

$\sum_{k}^{\infty} \sum_{l}^{\infty}\left[a_{m n k l}^{21} A_{k l}+a_{m n k l}^{22} B_{k l}+a_{m n k l}^{23} C_{k l}\right]=0$

$\sum_{k}^{\infty} \sum_{l}^{\infty}\left[a_{m n k l}^{31} A_{k l}+a_{m n k l}^{32} B_{k l}+a_{m n k l}^{33} C_{k l}\right]=0$

The solution of Eq. (19) leads to a thermal buckling criterion of the form

$$
\sum_{k} \sum_{l}\left[\frac{M_{m n k l}}{\Delta T}+P_{m n k l}+\delta_{m n k l}\right] A_{k l}=0
$$

in which $M_{m n k l}$ is the bending stiffness parameter, $P_{m n k l}$ is the extensional stiffness parameter, and $\delta_{m n k l}$ is a delta function dependent on the mechanical boundary conditions of the system. The buckling criterion can be evaluated with temperature-dependent material properties in an iterative process. For further details of the derivation, the reader is referred to Refs. 8 and 9.

This formulation provides a prediction of the critical temperature of the MFSPs studied in the present work. When evaluated for a square panel with clamped boundary conditions, the theoretical prediction is shown in Table 2 to be very similar to the experimentally measured values for the onset of buckling.
The theory, in its original form, cannot be used to model the preor postbuckling response measured in the present investigation for two reasons. First, the theory assumes rigid in-plane boundary conditions and, therefore, a null strain state in the prebuckling regime. To model the prebuckling behavior measured in the experiments, the analysis would require elastic in-plane boundary conditions. Second, the theory is a linear strain theory and, therefore, cannot predict a postbuckling response. A nonlinear extension of this theory and the inclusion of elastic in-plane boundary conditions are required to apply this theory to the prebuckling and postbuckling response of the panel.

\section{Conclusions}

The response of MFSPs to thermally induced equibiaxial in-plane loading has been investigated experimentally. The results are intended to provide experimental data that complement the relatively extensive theoretical and numerical investigations on the subject matter. Measurements have been made of the elastoplastic in-plane and out-of-plane response of MFSPs in the pre- and postbuckling regimes. The first critical buckling temperature has been measured and compared favorably to existing theory. The onset and evolution of the buckled mode shapes have been measured through shadow moire interferometry. The images, coupled with strain-gauge measurements, provide a useful illustration of the in-plane and outof-plane response of MFSPs to thermal loading up to and beyond critical design parameters. The experimental results have been obtained with a novel technique, designed to circumvent the problems commonly encountered with experimental thermomechanical boundary conditions. Validation of the thermal loading technique was achieved by comparing the experimental results for a solid thin aluminum plate and the theoretical results from classical thin-plate buckling theory. An understanding of the thermal buckling behavior of MFSPs is critical to the development of these panels for actively cooled TPSs. The experiments presented in the present paper provide a foundation for experiments on actively cooled inconel foam sandwich panels investigated by the present authors (see Ref. 19).

\section{Acknowledgment}

This research has been financially supported by NASA University Research Engineering and Technology Institute Award NCC 3989 through NASA Marshall Space Flight Center and NASA John H. Glenn Research Center at Lewis Field.

\section{References}

${ }^{1}$ Scotti, S. J., "Thermal Protection Systems for Reusable Launch Vehicles," Symposium on Structures for Hypersonic Vehicles, American Society of Mechanical Engineers, New York, Nov. 2003.

${ }^{2}$ Kelly, H. N., and Blosser, M. L., "Active Cooling from the Sixties to NASP," NASA CP-3157, 1992, pp. 189-249.

${ }^{3}$ Ellis, D. A., Pagel, L. L., and Schaeffer, D. M., "Design and Fabrication of a Radiative Actively Cooled Honeycomb Sandwich Structural Panel for Hypersonic Aircraft,” NASA CR-2957, 1978.

${ }^{4}$ Rakow, J. F., and Waas, A. M., "Size Effects and the Shear Response of Aluminum Foam," Mechanics of Materials, Vol. 37, No. 1, 2005, pp. 69-82.

${ }^{5}$ Rakow, J. F., and Waas, A. M., "Size Effects in Metal Foam Cores for Sandwich Structures," AIAA Journal, Vol. 42, No. 7, 2004, pp. 1331-1337.

${ }^{6}$ Rakow, J. F., and Waas, A. M., "The Effective Isotropic Moduli of Random Fibrous Composites, Platelet Composites, and Foamed Solids," Mechanics of Advanced Materials and Structures, Vol. 11, No. 2, 2004, pp. 151-173.

${ }^{7}$ Incropera, F. P., and DeWitt, D. P., Fundamentals of Heat and Mass Transfer, 5th ed., Wiley, New York, 2002, Chap. 6.

${ }^{8}$ Ko, W. L., "Mechanical and Thermal Buckling Analysis of Sandwich Panels Under Different Edge Conditions," NASA TM-4535, Jan. 1993.

${ }^{9}$ Ko, W. L., "Predictions of Thermal Buckling Strengths of Hypersonic Aircraft Sandwich Panels Using Minimum Potential Energy and Finite Element Methods," NASA TM-4643, May 1995.

${ }^{10}$ Noor, A. K., and Peters, J. M., "Postbuckling of Multilayered Composite Plates Subjected to Combined Axial and Thermal Loads," Thermal Structures and Materials for High-Speed Flight, edited by E. A. Thornton, Vol. 140, Progress in Aeronautics and Astronautics, AIAA, Washington, DC, 1992, pp. 183-203. 
${ }^{11}$ Hause, T., Librescu, L., and Johnson, T. F., "Non-Linear Response of Geometrically Imperfect Sandwich Curved Panels Under Thermomechanical Loading," International Journal of Non-Linear Mechanics, Vol. 33, No. 6, 1998, pp. 1039-1059.

${ }^{12}$ Javaheri, R., and Eslami, M. R., "Thermal Buckling of Functionally Graded Plates Based on Higher Order Theory," Journal of Thermal Stresses, Vol. 25, No. 7, 2002, pp. 603-625.

${ }^{13}$ Shen, H. S., "Thermomechanical Post-Buckling Analysis of Imperfect Laminated Plates Using a Higher-Order Shear-Deformation Theory," Computers and Structures, Vol. 66, No. 4, 1998, pp. 395-409.

${ }^{14}$ Blosser, M. L., "Boundary Conditions for Aerospace ThermalStructural Tests," Aerospace Thermal Structures and Materials for a New Era, edited by E. A. Thornton, Vol. 168, Progress in Aeronautics and Astronautics, AIAA, Washington, DC, 1995, pp. 119-144.

${ }^{15}$ Richards, W. L., and Thompson, R. C., "Titanium Honeycomb Panel Testing," NASA TM-4768, Oct. 1996.
${ }^{16}$ Ashby, M. F., Evans, A. G., Fleck, N. A., Gibson, L. J., Hutchinson, J. W., and Wadley, H. N. G., Metal Foams: A Design Guide, ButterworthHeinemann, Boston, 2000, Chap. 4.

${ }^{17}$ Singer, J., Arbocz, J., and Weller, T., Buckling Experiments: Experimental Methods in Buckling of Thin-Walled Structures, Vol. 1, Wiley, New York, 2002.

${ }^{18}$ Theocaris, P. S., Moire Fringes in Strain Analysis, Pergamon, New York, 1969.

${ }^{19}$ Rakow, J. F., "Thermomechanical Response of Metal Foam Sandwich Panels for Structural Thermal Protection Systems in Hypersonic Vehicles," $\mathrm{Ph} . D$. Dissertation, Dept. of Aerospace Engineering, Univ. of Michigan, Ann Arbor, MI, 2005.

${ }^{20}$ Reddy, J. N., Mechanics of Laminated Composite Plates, CRC Press, New York, 1997.

G. Agnes Associate Editor 